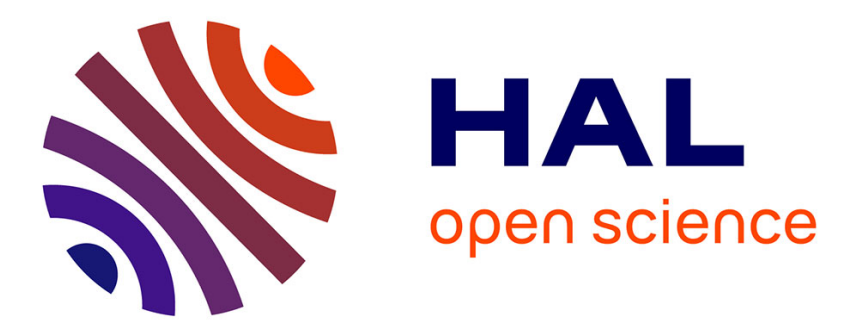

\title{
Low cost command device for single-phase induction motors
}

\author{
Frédéric Ferreyre, Guy Clerc, René Goyet, Thierry Bouscasse
}

\section{To cite this version:}

Frédéric Ferreyre, Guy Clerc, René Goyet, Thierry Bouscasse. Low cost command device for singlephase induction motors. SPEEDAM 2008, Jun 2008, Ischia, Italy. pp.278 - 283, 10.1109/SPEEDHAM.2008.4581141 . hal-00369566

\section{HAL Id: hal-00369566 https://hal.science/hal-00369566}

Submitted on 3 Jun 2009

HAL is a multi-disciplinary open access archive for the deposit and dissemination of scientific research documents, whether they are published or not. The documents may come from teaching and research institutions in France or abroad, or from public or private research centers.
L'archive ouverte pluridisciplinaire HAL, est destinée au dépôt et à la diffusion de documents scientifiques de niveau recherche, publiés ou non, émanant des établissements d'enseignement et de recherche français ou étrangers, des laboratoires publics ou privés. 


\title{
Low cost command device for single-phase induction motors
}

\author{
F. Ferreyre*, G. Clerc*, R.Goyet*, and T. Bouscasse** \\ * Université de Lyon, Lyon, F-69622, France ; université Lyon 1, Lyon, F-69622, France ; CNRS, UMR5005, Laboratoire \\ AMPERE, Villeurbanne, F-69622, (France) \\ ** Profalux Industrie ; 377 Rue des Cyprès, ZI Les Lanches, F-74300, Thyez, (France)
}

\begin{abstract}
The single-phase capacitor-run induction motor is widespread for small power applications where the price is an important concern. In order to save costs, one tries to estimate the speed on the basis of voltage and current measurements and thus avoid a speed sensor. This paper deals with the rotor speed measurement on the basis of the capacitor voltage and proposes a low cost command device for a rolling shutter induction motor.

First of all the article shows the analytical expression of the capacitor voltage as a function of the speed.

The suggested control device implements a microcontroller which cuts off the supply as soon as an abnormal reduction in speed occurs. The article details the implementation on the industrial application.
\end{abstract}

Index Terms-Capacitor motors, Induction motors, Sensorless speed Estimation, Microcontrollers.

\section{INTRODUCTION}

$\mathrm{C}$ APACITOR-run Single-Phase Induction Machines (SPIM) are very widespread in low cost drives supplied by the electric network: pumps, fans, lawn mowers, home automation, etc... In this frame, it often appears necessary to know the speed from electric measurements. The objectives are based on :

- driving requirements like the starting capacitor command [1] or vector controls [2]

- safety constraints like operating at excessive load, jamming or blockage. For instance, on rolling shutters, the arrival of the shutter at the buffer stop results in a drop in motor speed.

For compactness and low cost requirements the command circuit must need few electric sensors and small processing means. The solution is to research into the parameters which vary with the speed. One possible method is to measure the amplitude of the capacitor voltage in steady state operation.

The present study uses the terms of the impedance matrix and leads to the identification of the model parameters of an existing SPIM, using a genetic algorithm. The capacitor voltage curve versus the speed shows that this parameter is a representative function of the speed in steady state. A strategy is suggested to detect the slowing-down of the motor with few mathematical operations. This strategy is carried out with the early detection of the arrival of a rolling shutter at the buffer stop. The industrial reliability of the device is improved by a statistical approach in the determination of the slowdown thresholds.

\section{MODELING}

The two windings of the motor (subscript ' 1 ' and '2') and the capacitor $C$ are often supplied from the mains with a circuit comprising two switches $S w 1$ and $S w 2$ (Fig. 1). Closing one or the other of the two switches regulates the rotational direction. The switches are often placed at the bottom on the neutral side (point C). This configuration is interesting when using solid state switches like triacs, because the command of the gates is simple and does not require isolation device like optocouplers or transformers.

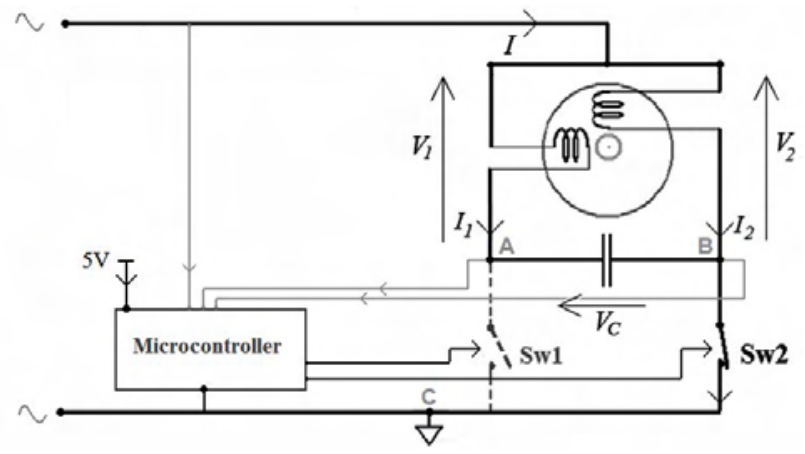

Fig.1. Supply device of a capacitor-run single-phase induction motor. The switches $S w 1$ and $S w 2$ allow to regulate the rotational direction.

The modeling considers that the two windings are identical (two-phase symmetrical induction machine) and does not take the iron losses into consideration. In these conditions SPIM may be represented by only two inductances, $L_{S}$ and $N$, and two resistances, $R_{S}$ and $R_{R}$.

The study of the motor, in unbalanced steady state sinusoidal regime, is made with Forward and Backward components. The voltages and currents of the stator are recorded with the indices 1 and 2, and the Forward and Backward symmetrical components with the exponents ${ }^{+}$ and - With the complex representation [5] the norm of the numbers represents the amplitude of the figures and not their RMS value.

This work was supported by the company Profalux Industrie. 
Calculations are turned to express the capacitor voltage between the two windings, 1 and 2, according to the rotor speed $\omega_{R}$. Conversely $\omega_{R}$ can be estimated from the measurement of the capacitor voltage. The equations for the currents are :

$$
\begin{aligned}
& \left(\begin{array}{l}
I_{1} \\
I_{2}
\end{array}\right)=\left(\begin{array}{cc}
1 & 1 \\
-j & j
\end{array}\right) \cdot\left(\begin{array}{l}
I^{+} \\
I^{-}
\end{array}\right) \\
& \left(\begin{array}{l}
I^{+} \\
I^{-}
\end{array}\right)=\frac{1}{2}\left(\begin{array}{cc}
1 & j \\
1 & -j
\end{array}\right) \cdot\left(\begin{array}{l}
I_{1} \\
I_{2}
\end{array}\right)
\end{aligned}
$$

and the same for the voltages.

The direct impedance $Z^{+}$and the inverse impedance $Z$ can be expressed by the equivalent dipoles of Fig. 2.a and 2.b [5], in which the slip $s=1-\frac{\omega_{R}}{\omega}$ is a function of the pulsation $\omega$ of the stator currents and the rotor speed $\omega_{R}$.

(a)

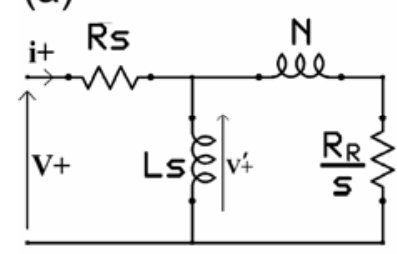

(b)

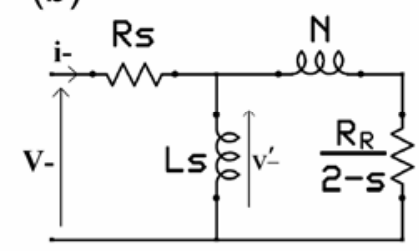

Fig. 2. Equivalent dipoles for forward (a) and backward (b) components temperature:

$R_{S}=275,305$ or $337 \Omega$ at 25,50 or $92^{\circ} \mathrm{C}$.

Then the iron losses are significant.

We have however adopted the four parameter model as it is practically analytical and makes it easier to represent in a fairly accurate manner the various operational regimes.

The four motor parameters were identified by an optimisation method involving the use of a genetic algorithm. The identification method (Fig. 3) consists in comparing the outputs of the analytic model with the real outputs measured on the motor. The optimisation algorithm adapts the model parameters so as to minimise the quadratic output error. After many iterations the numeric model approaches finely the true process.

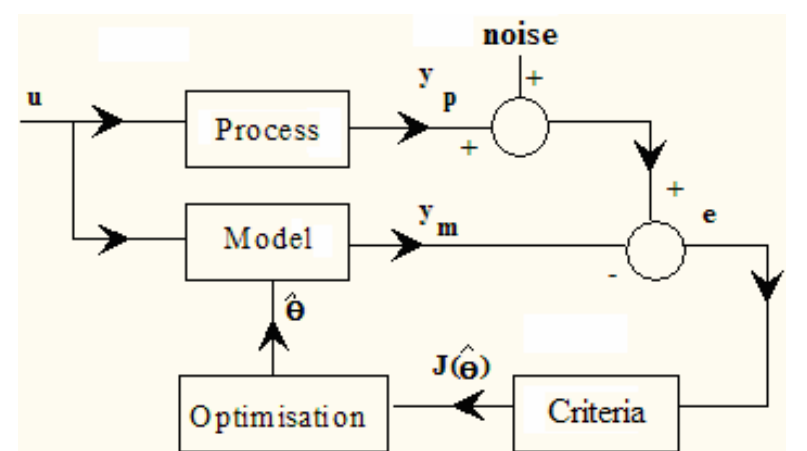

Fig 3. Identification method, minimization of the error between the real and the modeled process.

The real voltages and currents supplying the motor $\left(V_{1}, V_{2}, I_{1}, I_{2}\right)$ are sampled and recorded on a computer. The recorded samples of the mains voltage $V_{2}$ are applied as an input for the analytic model of the SPIM including the capacitor in steady state. $V_{l}$ is calculated using equations (6) and (7), and the currents are calculated in a similar way. The criterion is set up using the quadratic difference between the measured and calculated values in steady state.

The multimodal genetic algorithm described in [12] decides at each iteration step to modify the parameters of the analytic model (optimisation). Motor models are generally non-linear due to the variation of the speed. Genetic algorithms are well adapted to the identification of electric motors, for that they are not trapped by the local optima generated by the non-linearity. They converge to the global optima of the error function, providing the true parameters of the machine.

The parameter identification leads to the values :

$L_{S}=1.53 \mathrm{H}, N=0.072 \mathrm{H}, R_{S}=275 \Omega, R_{R}=475 \Omega$, the value of the capacitor $C$ being $4 \mu \mathrm{F}$.

\section{MOTOR CHARACTERISTICS}

Fig. 4 confirms the variation of the amplitude of the capacitor voltage with respect to the speed, as shown in equation (7). For our motor, this variation is monotonic and each value can be used to assess the relative speed $x$. 


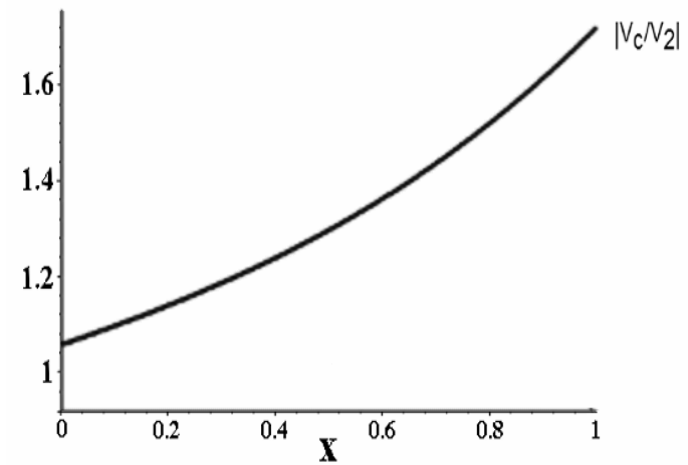

Fig.4. Amplitude of $V_{C} / V_{2}$ depending on the reduced speed

$$
x=\frac{\omega_{R}}{\omega}=1-s
$$

Other electrical parameters, like the phase difference between $V_{1}$ and $V_{2}$ or the ratio of their amplitude, can be relevant for estimating the speed as well. Indeed, in the complex plane (Fig. 5) the three voltages $V_{2}=O A, V_{1}=O C$ and $V_{C}=\mathrm{CA}$ form a triangle $O A C$ which is distorted when the relative speed $x$ varies. Fig. 5 gives the configuration of the voltages triangles obtained with our motor at two different speeds: $x=0$ (notation ') and $x=1$ (notation without').

This distortion depends on the motor and makes the measurement of some electrical parameters more relevant than others for the estimation of the speed.

For example, in the case of the rolling shutter SPIM of this paper, Fig 5 shows that $\left|V_{1}\right|=O C$, as well as $\left|V_{C}\right|=A C$, increases uniformly when the speed $x$ increases. The same results are obtained with the angle $(A O C)=\arg \left(V_{1} / V_{2}\right)$.

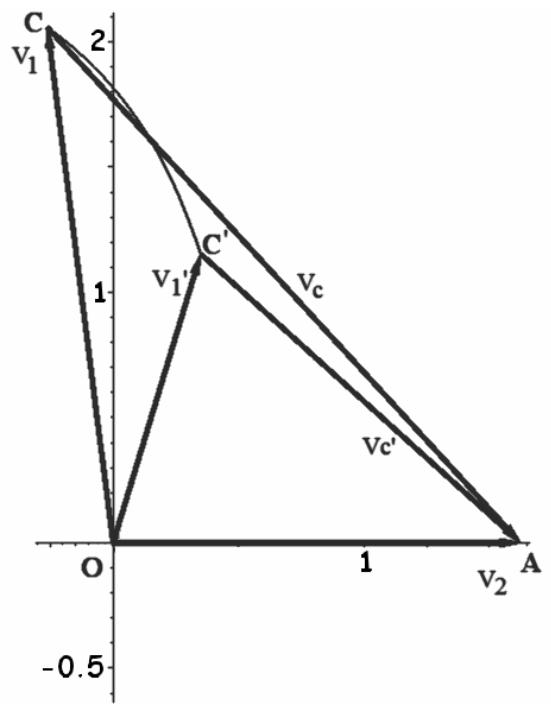

Fig. 5. Fresnel diagram of the stator voltages for $\mathrm{x} \in[0 ; 1]$ with their trajectories. Scale: " 1 " means $200 \mathrm{~V}$.

The vectors at the synchronism speed $(x=1)$ are noted without an asterisk $(\mathrm{X})$ and those of null speed $(x=0)$ are noted with an asterisk $\left(\mathrm{X}^{\prime}\right)$
On the contrary the angle $(C A O)$ changes from 47 degrees for $x=1$ to 42 degrees for $x=0$. It does not therefore vary a lot, and its measurement is not advisable for an estimation of $x$.

The electrical parameter that is the most suitable for the command circuit shown in Fig 1 is nevertheless $\left|V_{C}\right|=A C$ (or $\left|V_{C} / V_{2}\right|=A C / 230 \sqrt{2}$ ) because when one of the switches is closed this voltage is easily measurable with non-insulated voltage sensors referenced to the ground (point $\mathrm{C}$ ) and connected to the microcontroller (Fig 1). $V_{C}$ is indeed the voltage of the triac that remains opened.

\section{SLOWING DETECTION}

The previous study has shown that for rolling shutter SPIM the speed could be determined on the basis of $\left|V_{C} / V_{2}\right|$. It is adapted since, as seen in Fig. 4 this parameter decreases if the speed decreases. Therefore it is used to detect an excessive decrease in speed and to cut off the supply of the motor.

In this application, the decrease represents the arrival of the rolling shutter on the buffer stop. A signal processing is applied to the measurement " $y$ " of $\left|V_{C} / V_{2}\right|$ at each time step $\Delta t=10 \mathrm{~ms}$, and decides whether to stop the motor on not. The signal processing comprises three stages:

\section{A. Noise reduction}

At each $y_{i}$ three values are calculated:

the sliding average $S y_{i}=\left(y_{i}+y_{i-1}\right) / 2$,

the difference $D y_{i}=\left|y_{i}-y_{i-1}\right| / 2$,

and the thickness $E y_{i}=\max \left(D y_{i}\right)$.

These three values are a function of the evolution of $y$ and the background noise. They are used to calculate two envelopes, EnvMax and EnvMin, being the lower and the upper bound of $y_{i}$. The calculation is made according to :

If $S y_{i}>E n v M a x_{i}$ then :

$\left\{\right.$ EnvMax $_{i+1}=S y_{i}$ and EnvMin $\left.{ }_{i+1}=S y_{i}-E y_{i}\right\}$.

If $S y_{i}<E_{n v M i n}$ then :

$\left\{\right.$ EnvMin $_{i+1}=S y_{i}$ and EnvMax $\left.x_{i+1}=S y_{i}+E y_{i}\right\}$.

Fig. 6 shows how EnvMin ${ }_{i}$ and EnvMax ${ }_{i}$ surround $y$. During a continuous decrease in $y$, EnvMin $_{i}$ follows $S y_{i}$ which is itself very near $y_{i}$.

Once initialized in the first measurements the thickness $E y_{i}$ hardly changes at all in practice. Eventually, the system operates as a hysteresis follower with two outputs, one being the limit inferior and the other the limit superior of $y$. The outputs more or less follow $y$ by eliminating the noise. In the next stage, study of the variations of $y$ is carried out on the basis of EnvMin $_{i}$. 


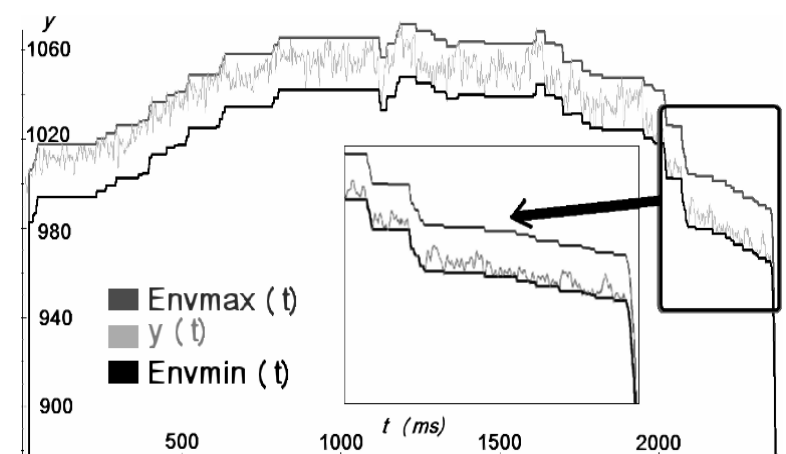

Fig. 6. Input signal and output envelopes for the noise reduction stage - (Env max) and (Env min) surround $y ; y$ : Arbitrary vertical scale -

\section{B. Buffering and detection}

The reduction in speed is studied from $N$ successive measurements of $y$ stored in the form of

$$
\alpha_{i} \cdot E n v \operatorname{Min}_{i}
$$

with $\alpha_{i}=1$ if $y$ decreases, i.e. if $\left(S y_{i}<E n v M i n_{i-1}\right)$, and $\alpha_{i}=0$ in the opposite case.

This algorithm takes into account only decreasing measurements of $y_{i}$. This has turned out to be beneficial in the case of rolling shutters to reduce calculation time and eliminate the irregularities of speed.

After each EnvMin ${ }_{i}$ storage, $N$ comparisons of $\alpha \cdot$ EnvMin to $N$ different thresholds $S(j)$ are made.

The motor is stopped if the following condition is verified:

$$
\left(\alpha_{i-j} \text { EnvMin }_{i-j}-\text { EnvMin }_{i}\right)>S_{j} .
$$

The comparison is not made if $\alpha_{i-j} E_{n v M i n} \operatorname{Mi-j}=0$. Equation (12) formulates the comparison between a threshold and the variation of Envmin through the elapsed time j. $\Delta t$.

Fig. 7 shows the process, with a 13 cell storage memory represented. At the fourth calculation, the difference 24 , is higher than the threshold 20 , the process is stopped and the motor turned off.

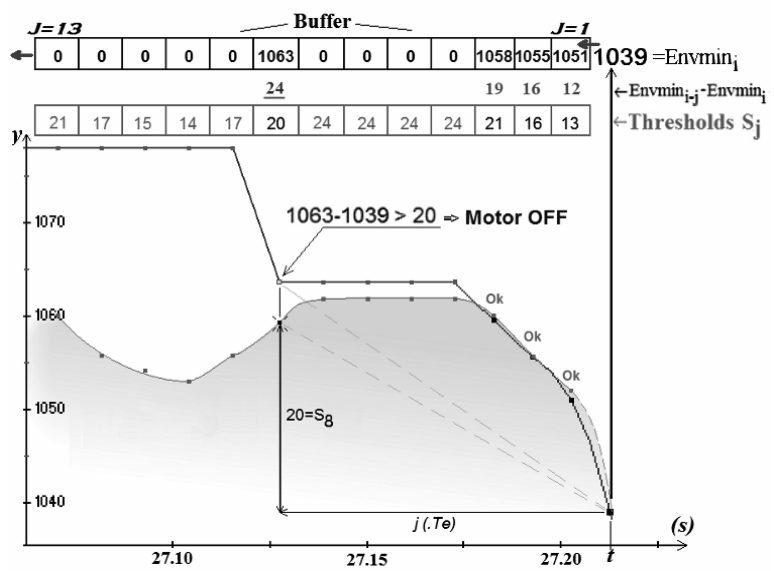

Fig.7. Multiple thresholds comparison ; $y$ : Arbitrary vertical scale -

\section{Threshold monitoring}

When the speed includes a low frequency component associated with a load irregularity, it is advisable to adapt the series of thresholds $S(j)$. The procedure can be made automatically thanks to a threshold controller which analyzes the speed variations, and decides consequently, to load a set of pre-calculated $N$ thresholds rather than another.

In the case of rolling shutters, we adopted $M=4$ sets of $N=18$ thresholds. Every $360 \mathrm{~ms}$ the series of $N$ EnvMin $_{i}$ is analyzed and the set of $N$ thresholds is changed if necessary.

\section{INDUSTRIAL APPLICATION ON A ROLLING SHUTTER}

\section{A. Equipment}

The previous study is implemented in practice to detect the arrival of a rolling shutter on the buffer stop, without sensor. The experimental bench is made up of a SPIM supplied by the AC power with an inversion device for the rotational direction (Fig. 1) made up of two triacs BT137B800F. The command shown in Fig. 8 is made around a microcontroller Microchip PIC16F886, 8 bits, 8 $\mathrm{MHz}, 512$ bytes of RAM, 256 bytes of ROM, CAN 10 bits. The microcontroller decodes the orders sent by radio by the user.

Two circuits (sensors) convert and filter the voltages of the triacs to $0-5 \mathrm{~V}$ compatible levels, and are connected to the two analog inputs on the microcontroller. The voltage of the open triac is $V_{C}$.

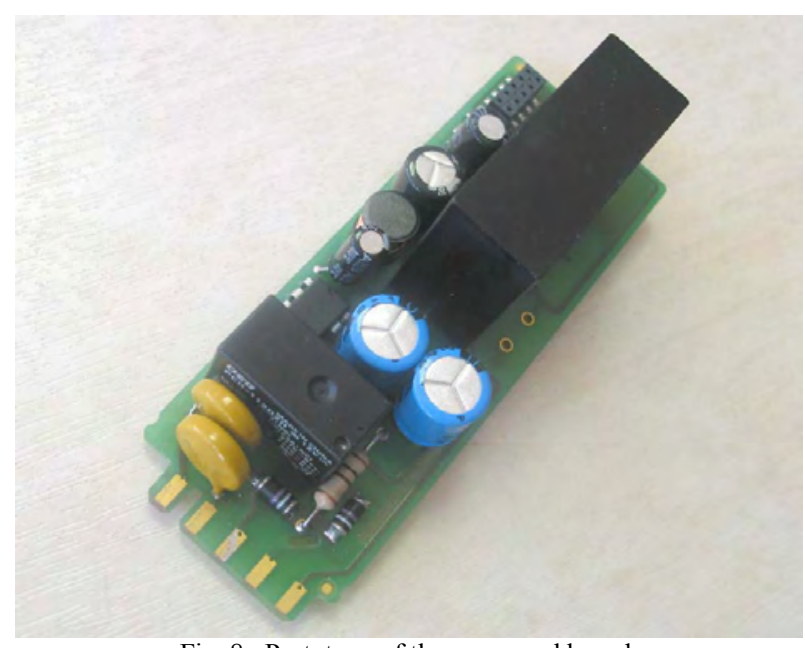

Fig. 8. Prototype of the command board.

\section{B. Statistical analysis and threshold determination}

The efficiency of the slowing-down detection algorithm strongly depends on the threshold values. These are determined on the basis of a statistical study of measurements, especially $V_{C}$ and the rotor speed $\mathrm{Nr}$, performed on many rolling shutters during operation. The programmed thresholds must be calculated so that they encompass all the possible configurations of rolling shutters, without adjustment nor reprogramming of the command board. 
For each recorded course of the shutter (test), during the normal operating range and before the drop in motor speed, a reciprocal algorithm provides the $N \times M$ minimum thresholds required for the system not to stop untimely. For each rank $n$ and set $m$ of threshold $S(n, m)$, the mean $\mu_{S}(n, m)$ and the standard deviation $\sigma_{S}(n, m)$ are calculated upon the population of tests. The assumptions are that the population of tests is large enough to be a representative sample of the total real population, and that the threshold population follows a normal distribution as a first approach.

The thresholds will be programmed following the equation

$S(n, m)=\mu_{S}(n, m)+k \times \sigma_{S}(n, m)$.

$k$ is the arbitrary margin, or the half-length of the confidence interval that should be considered so that the probability of spurious stoppage is less than $1 / 10000$. According to the normal distribution law, one can find : $k \geq 3.62$. The profiles of thresholds adopted for the industrial realization are given in Fig 9.

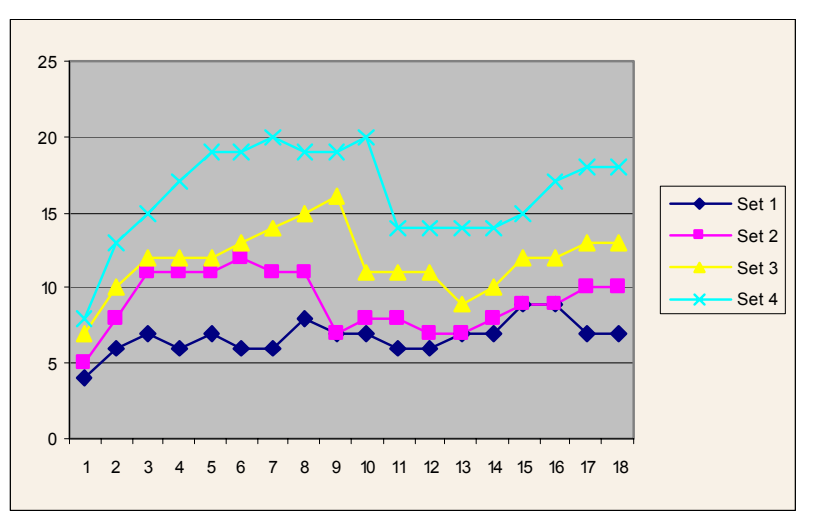

Fig.9. Profiles of thresholds adopted for the rolling shutter SPIM $(k=4)$ The set $\mathrm{n}^{\circ} 1$ is the most sensitive to variations of the speed, and the set $\mathrm{n}^{\circ} 4$, loaded by default at the motor start, is the most tolerant.

\section{Results}

So as to validate our approach, we have read the speed and the amplitude of $V_{C}$ at the time of a forced stoppage. The microcontroller detection is "off", and so the whole slowing-down process can be studied. The speed is measured by an incremental encoder giving one pulse per revolution. The slowing-down starts a little after $t_{1}=25.1 \mathrm{~s}$. The last speed measured $N_{2}$ is at $t_{2}=25.4 \mathrm{~s}-$ beyond this, accuracy is no longer ensured.

Fig. 10 illustrates clearly that the amplitude of $V_{C}$ has the same characteristics as the speed, with a measurement delay of 10 to $30 \mathrm{~ms}$. The theoretical results of paragraph II are confirmed.

On the recording, with the microcontroller detection "on", the application of the slowing detection algorithm with the most sensitive series of the 4 thresholds series, would have stopped the motor in $t_{S}=25290 \mathrm{~ms}$, i.e. approximately $60 \mathrm{~ms}$ after the beginning of the slowingdown. Experimentally this result is satisfactory. It makes it easier to clamp the shutter on the buffer stop without mechanical distortion nor audible grating.

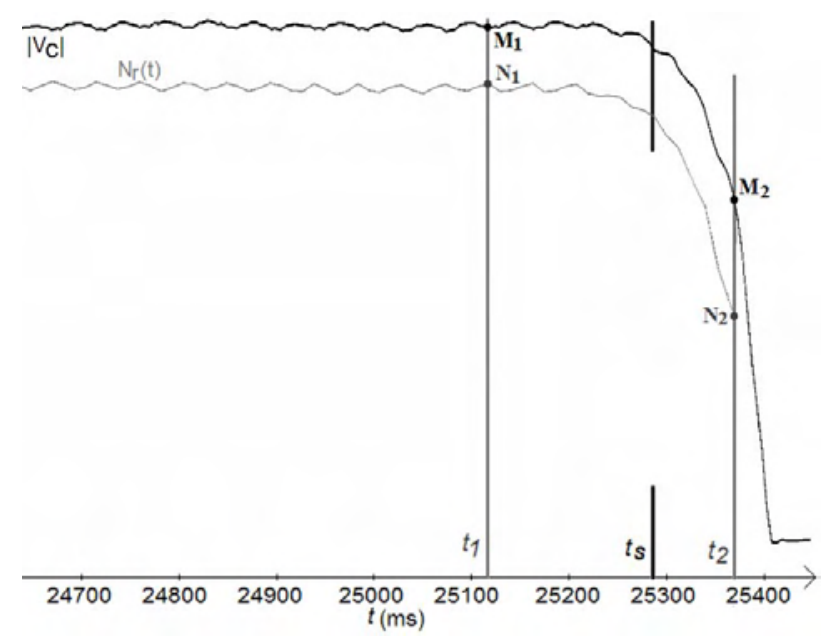

Fig. 10. Speed and capacitor voltage $V_{C}(t)$, for blocking the shutter when lowering without cut-out.

TABLE I

CHARACTERISTIC POINTS OF FIG. 9

\begin{tabular}{|l|l|l|l|}
\hline Abscissa $\boldsymbol{t},(\boldsymbol{m s})$ & $\mathbf{t}_{\mathbf{1}}=\mathbf{2 5 1 2 0}$ & $\mathbf{t}_{\mathbf{S}}=\mathbf{2 5 2 9 0}$ & $\mathbf{t}_{\mathbf{2}}=\mathbf{2 5 3 7 0}$ \\
\hline Speed $\boldsymbol{N}_{\boldsymbol{r}}(\boldsymbol{t}),(\boldsymbol{r p m})$ & $\mathrm{N}_{1}=2678$ & 2560 & $\mathrm{~N}_{2}=1808$ \\
\hline$\left|\boldsymbol{V}_{\boldsymbol{C}}\right|(\boldsymbol{t}),(\boldsymbol{V})$ & $\mathrm{M}_{1}=450$ & 439 & $\mathrm{M}_{2}=391$ \\
\hline
\end{tabular}

\section{CONCLUSION}

The article shows a system for assessing the speed and detecting the slowing down applied to the single phase induction motor with a running capacitor. The appraisal of the speed reposes on the measurement of the capacitor voltage. The system avoids the use of a speed sensor. The study starts by an analytic modeling of the motor in steady state. The parameters of the model are approached by an optimisation involving the use of a multimodal genetic algorithm.

The article suggests an algorithm which responds straight away to an excessive slowing-down while however remaining resistant to the electrical parasites and the irregularities of speed imposed by the load. The thresholds are finely adjusted to the load profile, by a statistical analysis of numerous measurements on the same family of loads, which makes it possible to anticipate the failure rate. Thus, the algorithm is suitably adaptable to any type of industrial application. The algorithm is implemented on a rolling shutter motor command device using a microcontroller, the objective being an end-of-travel stop. The measurement results are shown with related comments. 


\section{REFERENCES}

[1] J. P. Borrelli and R. Burkhart, "New phase-sensitive technology for capacitor start motor simplifies application", IEEE Trans. Industry Applications, vol. 34, no. 2, pp 253-257, March/April 1998.

[2] M. B. de Rossiter Corrêa, C. B. Jacobina, E. R. Cabral da Silva, A. M. Noguiera Lima, "Vector control strategies for single-phase induction motors drive systems", IEEE Trans. Industrial Electronics, vol. 51, no. 5, pp 1073-1080, October 2004.

[3] A. Payman, S. Vaez-Sadeh, "DSP based speed estimation of single phase induction motors", Power Electronics Specialists Conf., PESC 04. 2004 IEEE 35 ${ }^{\text {th }}$ Annual, vol. 2, pp 1335-1340.

[4] S. Vaez-Sadeh, S. H. Reicy, "Sensorless vector control of single-phase induction motor drives", Proc. of the $8^{\text {th }}$ IEEE Int. Conf. on Electrical Machines and Systems, 2005. ICEMS 2005, vol. 3, pp 1838-1842.

[5] P. Vas, "Electrical machines and drives, a space vector theory approach", Ed. Oxford, 1992, pp 502-511.

[6] H. Huang, E.F. Fuchs and J. C. White, "Optimal placement of the run capacitor in single phase induction motor design", IEEE Trans. Energy Conversion, vol. 3, no. 3, pp 647-652, September 1988.

[7] E. F. Fuchs, A. J. Vandenput, J. Höll and J. C. White, "Design and analysis of capacitor-start, capacitor-run single-phase induction motors", IEEE Trans. Energy Conversion, vol. 5, no. 2, pp 327-336, June 1990.

[8] L. Xu, "Dynamic model of an integrated-cycle controlled Single Phase Induction Machine" IEEE Trans. Energy Conversion, vol. 7, no. 4, pp 761-767, December 1992.

[9] Do-Hyun Jang, Jong-Soo Won, "Voltage, frequency, and phase-difference angle control of PWM inverters-fed twophase induction motors", IEEE Trans. Power Electronics, vol. 9, no. 4, pp 377-383, July 1994.

[10] C. Mademlis, I. Kioskeridis, T. Theodoulidis, "Optimisation of Single-Phase Motors, Part 1 : Maximum Energy Efficiency Control" IEEE Trans. Energy Conversion, vol. 20, no. 1, pp 187-195, March 2005.

[11] C. Mademlis, I. Kioskeridis, T. Theodoulidis, "Optimisation of Single-Phase Motors, Part 2 : Maximum Energy Efficiency Control", IEEE Trans. Energy Conversion, vol. 20, no. 1, pp 196-203, March 2005.

[12] G. Clerc, M. Bessaou, P. Siarry, P. Bastiani, "Identification des machines synchrones par algorithme génétique", RIGE vol. 5, no. 3-4, pp 485-515, April 2002. 